\title{
High attenuation areas on chest computed tomography in community- dwelling adults: the MESA study
}

\author{
Anna J. Podolanczuk ${ }^{1,17}$, Elizabeth C. Oelsner ${ }^{1,17}$, R. Graham Barr ${ }^{1,2}$, \\ Eric A. Hoffman ${ }^{3}$, Hilary F. Armstrong ${ }^{2}$, John H.M. Austin ${ }^{4}$, Robert C. Basner ${ }^{1}$, \\ Matthew N. Bartels ${ }^{5}$, Jason D. Christie ${ }^{6}$, Paul L. Enright", \\ Bernadette R. Gochuico ${ }^{8}$, Karen Hinckley Stukovsky ${ }^{9}$, Joel D. Kaufman ${ }^{10}$, \\ P. Hrudaya Nath ${ }^{11}$, John D. Newell Jr ${ }^{3}$, Scott M. Palmer ${ }^{12}$, Dan Rabinowitz ${ }^{13}$, \\ Ganesh Raghu $^{10}$, Jessica L. Sell ${ }^{1}$, Jered Sieren ${ }^{14}$, Sushil K. Sonavane ${ }^{11}$, \\ Russell P. Tracy ${ }^{15}$, Jubal R. Watts ${ }^{11}$, Kayleen Williams ${ }^{10}$, Steven M. Kawut ${ }^{16}$ and \\ David J. Lederer ${ }^{1,2}$
}

\begin{abstract}
Affiliations: ${ }^{1}$ Dept of Medicine, Columbia University Medical Center, New York, NY, USA. ${ }^{2}$ Dept of Epidemiology, Columbia University Medical Center, New York, NY, USA. ${ }^{3}$ Depts of Radiology, Medicine and

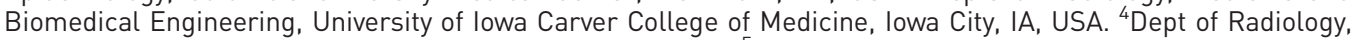
Columbia University Medical Center, New York, NY, USA. ${ }^{5}$ Dept of Rehabilitation Medicine, Albert Einstein College of Medicine/Montefiore Medical Center, Bronx, NY, USA. ${ }^{6}$ Dept of Medicine and the Center for Translational Lung Biology, University of Pennsylvania, Philadelphia, PA, USA. ${ }^{7}$ Dept of Epidemiology, University of Arizona, Tucson, AZ, USA. ${ }^{8}$ National Human Genome Research Institute, Bethesda, MD, USA. ${ }^{9}$ Dept of Biostatistics, University of Washington, Seattle, WA, USA. ${ }^{10}$ Dept of Medicine, University of Washington, Seattle, WA, USA. ${ }^{11}$ Dept of Radiology, University of Alabama, South Birmingham, AL, USA. ${ }^{12}$ Duke Clinical Research Institute, Duke University, Durham, NC, USA. ${ }^{13}$ Dept of Statistics, Columbia University, New York, NY, USA. ${ }^{14}$ VIDA Diagnostics, Inc., Coralville, IA, USA. ${ }^{15}$ Dept of Pathology, University of Vermont, Colchester, VT, USA. ${ }^{16}$ Dept of Medicine and the Center for Clinical Epidemiology and Biostatistics, Perelman School of Medicine at the University of Pennsylvania, Philadelphia, PA, USA. ${ }^{17}$ Both authors contributed equally to this work.
\end{abstract}

Correspondence: David J. Lederer, Medicine and Epidemiology, Columbia University Medical Center, 161 Fort Washington Ave, Room 3-321A, New York, NY 10032. E-mail: davidlederer@columbia.edu

ABSTRACT Evidence suggests that lung injury, inflammation and extracellular matrix remodelling precede lung fibrosis in interstitial lung disease (ILD). We examined whether a quantitative measure of increased lung attenuation on computed tomography (CT) detects lung injury, inflammation and extracellular matrix remodelling in community-dwelling adults sampled without regard to respiratory symptoms or smoking.

We measured high attenuation areas (HAA; percentage of lung voxels between -600 and -250 Hounsfield Units) on cardiac CT scans of adults enrolled in the Multi-Ethnic Study of Atherosclerosis.

HAA was associated with higher serum matrix metalloproteinase-7 (mean adjusted difference $6.3 \%$ per HAA doubling, 95\% CI 1.3-11.5), higher interleukin-6 (mean adjusted difference 8.8\%, 95\% CI 4.8-13.0), lower forced vital capacity (FVC) (mean adjusted difference $-82 \mathrm{~mL}, 95 \% \mathrm{CI}-119--44$ ), lower 6-min walk distance (mean adjusted difference $-40 \mathrm{~m}, 95 \% \mathrm{CI}-1--80$ ), higher odds of interstitial lung abnormalities at 9.5 years (adjusted OR 1.95, 95\% CI 1.43-2.65), and higher all cause-mortality rate over 12.2 years (HR 1.58, 95\% CI 1.39-1.79).

High attenuation areas are associated with biomarkers of inflammation and extracellular matrix remodelling, reduced lung function, interstitial lung abnormalities, and a higher risk of death among community-dwelling adults.

$@$ ERSpublications

Increased lung attenuation on CT may identify subclinical lung injury and inflammation in community-dwelling adults http://ow.ly/97k3300tvKX 


\section{Introduction}

Recurrent alveolar epithelial cell injury, lung inflammation, and dysregulated extracellular matrix remodelling are thought to be key precursor events that lead to lung fibrosis in interstitial lung disease (ILD) $[1,2]$. Adults with ILD typically develop symptoms years after the initial insult, making the antecedent causes of lung fibrosis difficult to identify. Studies of humans with subclinical disease may provide novel insights into the early mechanisms of lung injury; yet, until recently, few studies have looked at interstitial lung changes prior to the development of symptoms [3-5].

Recent recognition of interstitial lung abnormalities (ILA) on chest CT scans of participants in large population cohorts has advanced our understanding of early interstitial lung disease [6-9]. ILAs have been shown to be associated with cigarette smoking [6], reduced lung volumes [6], reduced exercise capacity [7], MUC5b polymorphism [8], and all-cause mortality [9] in population-based cohorts. However, ILA assessment is labour intensive, requiring visual scoring by trained readers, and may, at times, exclude equivocal and indeterminate findings that may have clinical significance. An automated approach might provide superior reproducibility and greater sensitivity for early abnormalities not detectable by the human eye, including changes that might precede visual identification of ILA.

We have previously proposed that a quantitative measure of lung attenuation on computed tomography (CT), termed high attenuation areas (HAA), might be a suitable measure of early pathological processes in the lung that precede ILD [10]. We reported that a greater volume of HAA in the lung is associated with cigarette smoking [10]. Others reported that adults with HAA involving more than $10 \%$ of the lung have reduced total lung capacity and an increased odds of ILA in cross-sectional analyses [11]. However, further construct validity of HAA as a quantitative measure of early lung injury, inflammation or fibrosis is lacking and the significance of smaller amounts of HAA is not known.

In the current study, we therefore sought to determine whether HAA is associated with features of subclinical lung injury, inflammation and fibrosis, including higher levels of serum biomarkers of inflammation (interleukin-6 (IL-6) and C-reactive protein (CRP)) and lung remodelling (surfactant protein-A (SP-A) and matrix metalloproteinase-7 (MMP-7)), reduced FVC, reduced exercise capacity, increased odds of visually-identified ILA, and all-cause mortality in community dwelling adults. We chose IL-6 and CRP because of their well-characterised roles in systemic inflammation [12, 13], and MMP-7 and SP-A based on prior studies showing associations of these biomarkers with lung injury and extracellular matrix remodelling in ILD [14-20].

\section{Methods}

Full methods are available in the supplementary appendix.

\section{Study participants}

The Multi-Ethnic Study of Atherosclerosis (MESA) is a multi-centre, National Heart Lung and Blood Institute (NHLBI)-sponsored US-based prospective cohort study designed to investigate subclinical cardiovascular disease. Enrolment criteria have previously been described [21]. Briefly, the study enrolled 6814 adults aged 45 and 84 years free of clinical cardiovascular disease from six communities in the USA between 2000 and 2002. There were no selection criteria based on lung disease, respiratory symptoms or smoking history. MESA participants attended follow-up visits, with the most recent visit between 2010 and 2012 (figure S1) [21]. Vital status was determined by contacting each participant or family member every 9-12 months. This was supplemented through review of the National Death Index (NDI) to ensure complete follow-up of mortality through the most recent NDI update (March 13, 2015).

MESA served as the sampling frame for our study. We sampled MESA participants for three substudies: biomarkers, lung function and ILA measurement, and exercise testing. Our sampling strategies for each substudy are described in detail in the supplementary material and in figure S2. The sample size for each substudy is reported alongside the results of each substudy.

This article has supplementary material available from erj.ersjournals.com

Received: Jan 162016 | Accepted after revision: May 072016 | First published online: July 282016

Support statement: This research was supported by grants and contracts R01 HL103676, R01 HL077612, R01 HL114626, RC1-100543 and R01-HL093081, N01-HC-95159, N01-HC-95160, N01-HC-95161, N01-HC-95162, N01-HC-95163, N01-HC-95164, N01-HC-95165, N01-HC-95166, N01-HC-95167, N01-HC-95168, N01-HC-95169, and T32 HL105323 from the National Heart, Lung, and Blood Institute, and by grants UL1-TR-000040 and UL1-RR-025005 from NCRR/NCATS. This project was funded in part by the Pulmonary Fibrosis Foundation. Funding information for this manuscript has been deposited with FundRef.

Conflict of interest: Disclosures can be found alongside the online version of this article at erj.ersjournals.com 
We also included a comparator group of 48 adults with clinically diagnosed ILD (75\% with idiopathic pulmonary fibrosis (IPF)) seen at one of three centres (Columbia, Penn, or Duke) who were enrolled in the NHLBI-funded Lung Transplant Body Composition (LTBC) study.

MESA, LTBC, and all ancillary studies were approved by Institutional Review Boards at all collaborating centres and all participants provided informed consent.

\section{High attenuation areas on CT scans}

HAA was measured on non-contrast cardiac CT scans performed at the MESA baseline visit in 2000-2002 on multi-detector CT scanners (three sites) or electron beam tomography scanners (three sites) using a previously described standardised protocol [22]. Each participant underwent two sequential scans on separate breath holds in succession at full inspiration, with transverse fields of view capturing the whole lung field from the carina to the lung bases. The scan with higher air volume was used for analyses, except in cases of discordant scan quality control score, in which case the higher quality scan was used [23]. Details of the CT protocols are shown in table S1 in the supplementary appendix. A prior validation study using MESA full-lung scans showed that these cardiac CT scans image approximately $65 \%$ of total lung volume, excluding most of the upper lobes but capturing most of the lower lobes [23]. HAA was defined as the percentage of imaged lung volume having CT attenuation between -600 and -250 Hounsfield Units (HU), as previously described [10]. Percentage emphysema was defined as the percentage of voxels below $-950 \mathrm{HU}$ [24]. LTBC participants underwent full lung inspiratory CT imaging according to clinical protocols at each site. Quantitative image attenuation was measured using a modified version of the Pulmonary Analysis Software by trained readers at the University of Iowa Imaging Lab (Iowa City, IA, USA).

\section{Biomarker measurements}

MMP-7, SP-A, and interleukin-6 (IL-6) were measured in banked baseline serum samples using ELISA assays from R\&D systems (MMP-7 and IL-6) and Biovendor (SP-A). Baseline serum C-reactive protein (CRP) was measured using a BNII nephelometer. All measurements were performed by the MESA Core Laboratory at the University of Vermont's Laboratory for Clinical Biochemistry Research (Burlington, VT, USA). Additional assay characteristics are provided in the supplementary appendix.

\section{Spirometry and exercise testing}

Spirometry was conducted according to the American Thoracic Society (ATS)/European Respiratory Society guidelines [25], as previously described [26]. 6-min walk test (6MWT) was conducted in accordance with ATS guidelines without supplemental oxygen [27]. CPET was performed on a Vmax Encore 29 Metabolic Exercise System, Viasprint 150P (Viasys Respiratory Care, Yorba Linda, CA, USA) with an electrically braked cycle ergometer.

\section{Interstitial lung abnormalities}

Full-lung MESA CT scans were acquired in years 2010-12 at suspended full inspiration on 64-slice scanners (GE and Siemens) using the MESA Lung/SPIROMICS protocol (See supplementary appendix) [28]. Images were reconstructed using $0.625 \mathrm{~mm}$ slice thickness, as previously described [29]. ILA was defined as the presence of ground-glass, reticular abnormality, diffuse centrilobular nodularity, honeycombing, traction bronchiectasis, non-emphysematous cysts or architectural distortion in at least 5\% of nondependent portions of the lung, using methodology previously described $[6,8,30]$.

\section{Analysis approach}

We treated HAA as a continuous independent log base-2 (binary) transformed variable. For most analyses, we used generalised linear models adjusted for age, sex, race/ethnicity, educational attainment, height, body mass index (BMI), waist circumference, smoking status, cigarette pack-years, glomerular filtration rate (GFR), study site, $\mathrm{mA}$ dose, total volume of imaged lung and percentage emphysema. Since FVC and imaged lung volume were collinear (Pearson correlation coefficient of 0.7), we excluded imaged lung volume from analyses examining the association between HAA and FVC. We used logistic regression to estimate adjusted odds ratios for ILA. Given the small sample size of the exercise substudy, we chose a case-control design. We defined HAA cases as having the highest decile of HAA $(>7.5 \%$ of total imaged lung) and we present age- and sex-adjusted least-square mean values. Details of sampling for analyses of MMP-7 and SP-A are provided in the supplementary appendix.

We examined associations between HAA and survival time using Cox proportional hazards models and additive Cox models, as previously described [31]. Survival time was calculated as the age at death or last follow-up or the most recent National Death Index update, whichever occurred later. Primary analyses were adjusted for the above covariates. Secondary analyses were further adjusted for alcohol use, exercise, history of cancer, coronary artery calcification and the cardiovascular risk factors listed in the footnote to table 3. 
We examined models stratified by smoking status, age, sex, race/ethnicity and BMI. We performed sensitivity analyses excluding participants in the highest decile of HAA ( $>7.5 \%$ of total imaged lung) and adjusting for measures of cardiac systolic and diastolic function. We compared mean HAA values among community-dwelling adults to HAA values in 48 adults clinically diagnosed with ILD using the Wilcoxon rank sum test.

All statistical tests were performed in SAS version 9.3 (SAS Institute) and R version 3.2.0 (R Foundation for Statistical Computing).

\section{Results}

One participant was excluded from analyses due to lack of valid baseline HAA assessment (figure S2). The baseline characteristics of the 6813 remaining MESA participants are shown in tables S2 and S3 in the supplementary appendix. MESA participants had a mean age of 62 years; 53\% were men; $38 \%$ were white, $28 \%$ were African-American, $22 \%$ were Hispanic and $12 \%$ were Asian. $55 \%$ of MESA participants were former or current smokers who smoked a median of 15 cigarette pack-years. The 48 LTBC participants had a mean age of 63 years (range $34-75$ years), 31\% were women and $75 \%$ had IPF.

MESA participants had a mean \pm SD (range) HAA value of $5.1 \pm 3.1 \%(1.2 \%-48.9 \%)$. LTBC participants with clinically diagnosed ILD had a mean (SD) HAA value of $25.4 \%(8.8 \%)$ with a range of $9.3 \%-50.8 \%$ (Wilcoxon rank sum $\mathrm{p}$-value $<0.001$ versus MESA participants; figure 1a). MESA participants with greater HAA were more likely to be female, Hispanic or Chinese, never-smokers, and had higher BMIs than those with lower HAA values (table S2). Those in the higher HAA quartiles also tended to have a lower volume of imaged lung and lower percent emphysema.

\section{Visual appearance of HAA on CT}

MESA participants with elevated HAA (defined arbitrarily as greater than $7.5 \%$ ) had a range of abnormalities on visual inspection of CT images [10]. Common abnormalities included ground glass and reticular changes. Figure 1 shows selected CT images from four MESA participants with HAA >90th percentile at baseline who also had ILA at 10-year follow-up.
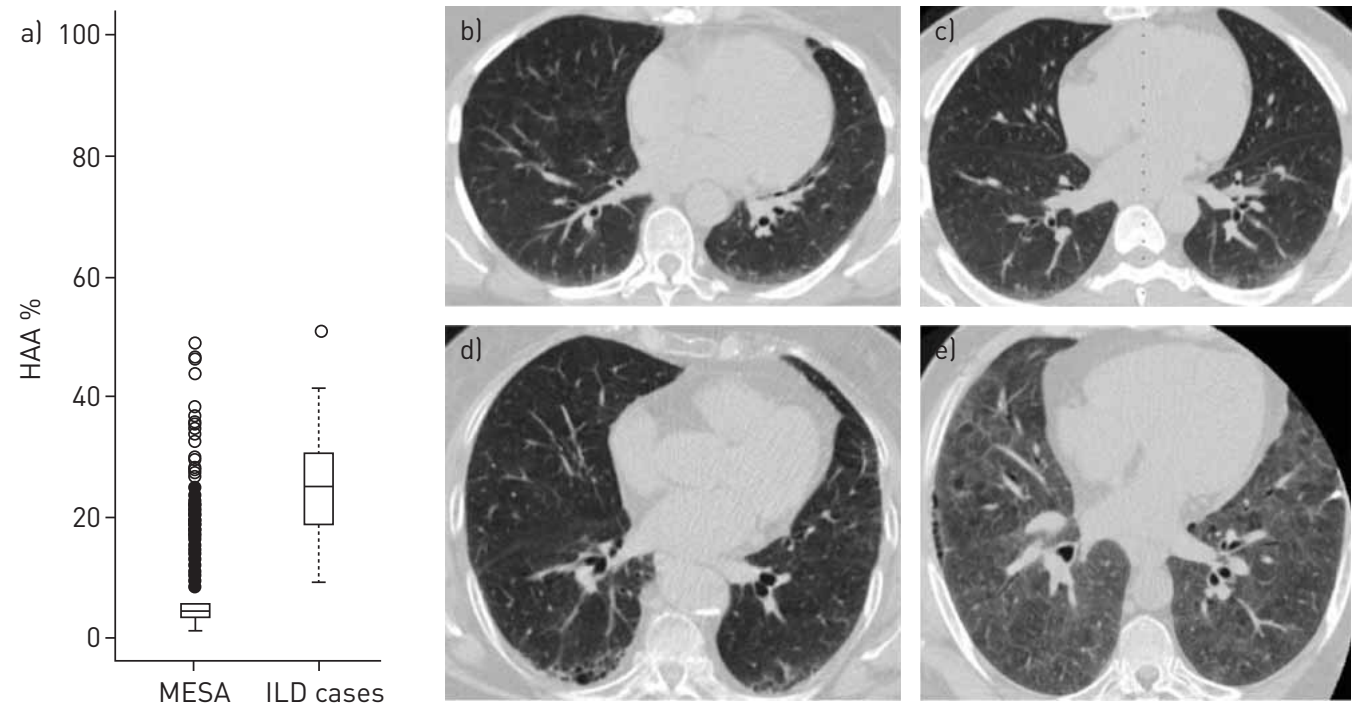

FIGURE 1 a) Boxplots of high attenuation area (HAA) values for 6813 Multi-Ethnic Study of Atherosclerosis (MESA) participants and 48 adults with clinically diagnosed interstitial lung disease enrolled in the Lung Transplant Body Composition Study. Solid horizontal lines represent the median HAA value. Boxes are bound by the upper and lower quartiles. Whiskers extend to 1.5-times the interquartile range. Outliers are represented by open circles. Wilcoxon rank sum test $p$-value $<0.001$. b-e) Selected computed tomography scan images from four MESA participants with elevated HAA. b) Peripheral basilar ground glass abnormality with $\mathrm{HAA}=8.8 \%$ of the imaged lung. c) Ground-glass opacity with $\mathrm{HAA}=10.0 \%$ of the imaged lung. d) Early peripheral reticular and cystic changes with $\mathrm{HAA}=10.2 \%$ of the imaged lung. e) Diffuse ground glass abnormality and an area of peripheral reticular changes with $\mathrm{HAA}=27.7 \%$ of imaged lung. All participants had interstitial lung abnormalities on follow-up computed tomography scans at MESA exam 5 (median follow-up time 9.5 years). None of the participants was clinically diagnosed with interstitial lung disease. 


\section{Serum biomarkers}

There were 908 MESA participants who had MMP-7 and SP-A measured (see supplementary appendix). IL-6 was measured in 6621 participants and CRP was measured in 6671 participants. Greater HAA was associated with higher serum MMP-7 and IL-6 levels (table 1 and table S4). For each doubling of HAA, there was a $6.26 \%$ increment in MMP-7 (95\% CI 1.29-11.47; $\mathrm{p}=0.01$ ) (figure 3a) and an 8.78\% in IL-6 (95\% CI 4.77-12.95; $\mathrm{p}<0.001$ ) (figure $3 \mathrm{~b}$ ). The association between HAA and MMP-7 was modified by smoking status $(\mathrm{p}<0.001$ for interaction). Each doubling in HAA was associated with a $15.45 \%$ increment in MMP-7 (95\% CI 7.91-23.53; p<0.001) among ever-smokers and a 1.39\% increase in MMP-7 (95\% CI -5.36 to $8.62 ; \mathrm{p}=0.69$; table 1 ) among never-smokers. The association between HAA and IL- 6 tended to be stronger among ever-smokers albeit with only modest evidence of effect modification $(\mathrm{p}=0.11$ for interaction; table 1). We found no significant association between HAA and CRP or SP-A levels (table 1).

\section{Spirometry}

There were 3834 MESA participants who completed spirometry and had had valid measures of forced expiratory volume in $1 \mathrm{~s}$ (FEV1) and FVC (see supplementary appendix). In a fully adjusted model, each doubling of HAA was associated with an $82 \mathrm{~mL}$ decrement in FVC (95\% CI 119-44; p <0.001) and a $2.8 \%$ absolute decrement in percentage predicted FVC (95\% CI 3.9-1.7; $\mathrm{p}<0.001$; table S5) in MESA. This association was modified by smoking status ( $\mathrm{p}=0.002$ for interaction). In a fully adjusted model, each doubling of HAA was associated with a $157 \mathrm{~mL}$ decrement in FVC (95\% CI 216-97; p <0.001) (table S5) among ever-smokers and a $15 \mathrm{~mL}$ decrement (95\% CI 60--31; p=0.53) (table S5) among never-smokers. We found similar associations for $\mathrm{FEV}_{1}$ and percent predicted FEV 1 but not for the ratio of FEV1 to FVC (table S6).

\section{Exercise capacity}

There were 89 MESA participants who completed exercise testing; 30 of these were cases (had HAA above the 90th percentile (7.5\%)) and 59 were controls (HAA below the 90th percentile). Cases with high HAA had diminished exercise capacity by both 6-MWT and CPET compared with controls (figure 2). After adjustment for age and sex, the least squares mean difference was $40 \mathrm{~m}$ (95\% CI 1-80; p=0.045; table S7). HAA cases also had lower adjusted peak oxygen uptake $\left(V^{\prime} \mathrm{O}_{2}\right)\left(1.28\right.$ versus $1.47 \mathrm{~L} \cdot \mathrm{min}^{-1}$; $\left.\mathrm{p}=0.01\right)$, percent predicted peak $V^{\prime} \mathrm{O}_{2}(72 \%$ versus $83 \% ; \mathrm{p}=0.01)$ and peak Watts $(76$ versus $88 \mathrm{~W} ; \mathrm{p}=0.02$; figure 2 and table S7). Breathing reserve was lower among cases than controls (53 versus 61\%; $\mathrm{p}=0.04$ ), suggesting a ventilatory limitation to exercise. There were no significant differences between cases and controls in heart rate, $\mathrm{O}_{2}$ pulse, minute ventilation/ $\mathrm{CO}_{2}$ production, and end-tidal $\mathrm{CO}_{2}$ at peak exercise.

\section{Interstitial lung abnormalities}

There were 2907 MESA participants who had a valid qualitative assessment of ILA. The median time between HAA measurement and ILA assessment was 9.5 years (range 8.0-11.4 years). Of these 2907 scans, 477 (16.5\%) were read as equivocal for ILA and were excluded from further ILA analyses. Of the remaining 2430 participants, 306 (10.5\% of the overall sample) had ILA: 285 (9.8\%) had scans read as suspicious for ILD and $21(0.7 \%)$ had scans showing bilateral fibrosis in multiple lobes associated with honeycombing and traction bronchiectasis in a subpleural distribution (definite ILD). 162 (53\%) participants with ILA had

TABLE 1 Cross-sectional associations between high attenuation areas (HAA) and serum biomarkers of lung injury, extracellular matrix remodelling and inflammation at the Multi-Ethnic Study of Atherosclerosis (MESA) baseline exam stratified by smoking status

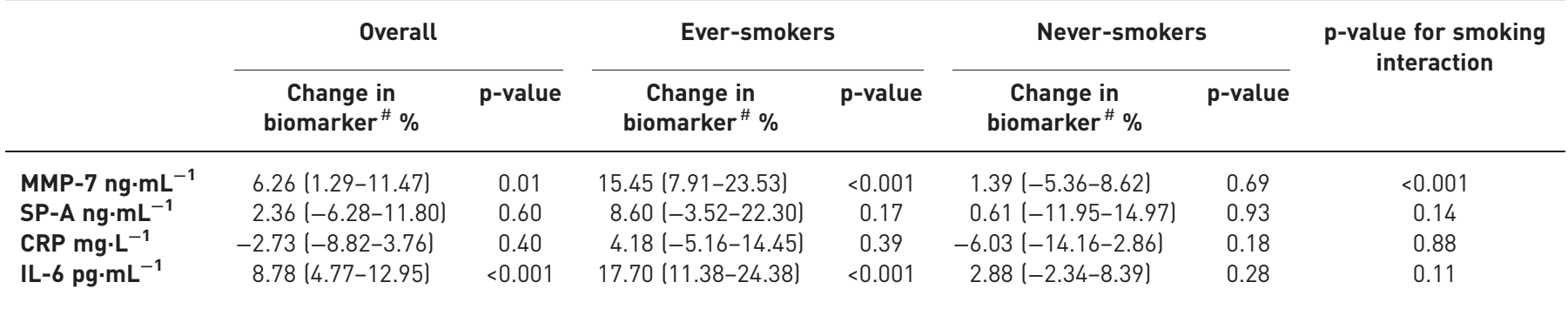

MMP-7: matrix metalloproteinase-7; SP-A: surfactant protein-A, CRP: C-reactive protein; IL-6: interleukin-6. For MMP-7 and SP-A, n=908 (341 cases defined as having HAA $>9 \%$ and a randomly selected subcohort of 600 MESA participants; 33 participants in the subcohort also met case definition); for CRP, $n=6761$; for IL-6, $n=6621$. \#: adjusted for age, sex, race/ethnicity, educational attainment, height, body mass index, waist circumference, smoking status, cigarette pack-years, glomerular filtration rate, study site, mA dose, total volume of imaged lung and percentage emphysema. Adjustment for smoking status and pack-years was excluded from smoking-stratified models. All covariates measured at baseline examination in 2000-2002. Percentage change is reported per two-fold increase in HAA. 
changes that were primarily in the subpleural distribution. The distribution of the types of abnormalities is shown in table S8 in the supplementary appendix.

The number of participants with ILA increased across quartiles of HAA ( $<<0.001$ for trend; table 2). In fully adjusted models, those with baseline HAA values greater than 5.3\% (4th quartile) had a three-fold higher odds of ILA compared with those in the lowest quartile (OR 3.07, 95\% CI 1.77-5.33; p<0.001; table 2 and figure 3c), and each doubling of HAA was associated with a 95\% increased odds of ILA (OR 1.95, 95\% CI 1.43-2.65, p<0.001). These findings were similar among ever-smokers and never-smokers (table 2). Analyses in which participants with equivocal ILA findings were included as "no ILA" yielded similar results (table S9).

\section{Mortality}

Five participants were excluded from follow-up by the MESA Events Committee because of discovery of pre-baseline cardiovascular events, and no mortality data are available for these participants. There were 1093 deaths among the remaining 6808 MESA participants over a median follow-up of 12.2 years (range 0-14.2). Greater HAA was associated with higher all-cause mortality (figure 3d). In fully adjusted models, MESA participants in the highest quartile of HAA had approximately a doubling of the rate of death compared with those in the lowest HAA quartile (HR 1.94, 95\% CI 1.53-2.46; $\mathrm{p}<0.001$ for trend; table 3) and each doubling of HAA was associated with a $55 \%$ increase in the rate of death from any cause (HR $1.55,95 \%$ CI 1.37-1.77; $\mathrm{p}<0.001$ ) without evidence of modification by smoking status.

\section{Additional analyses}

Stratified and sensitivity analyses, including adjustment for left ventricular function and restriction to those participants with HAA less than 7.5\%, are shown in tables S10-S12. These analyses did not substantially change our findings.

\section{Discussion}

We have shown that areas of high CT attenuation in the lungs of community-dwelling middle aged and older adults were associated with elevated serum levels of MMP-7 and IL-6, reduced FVC, reduced exercise
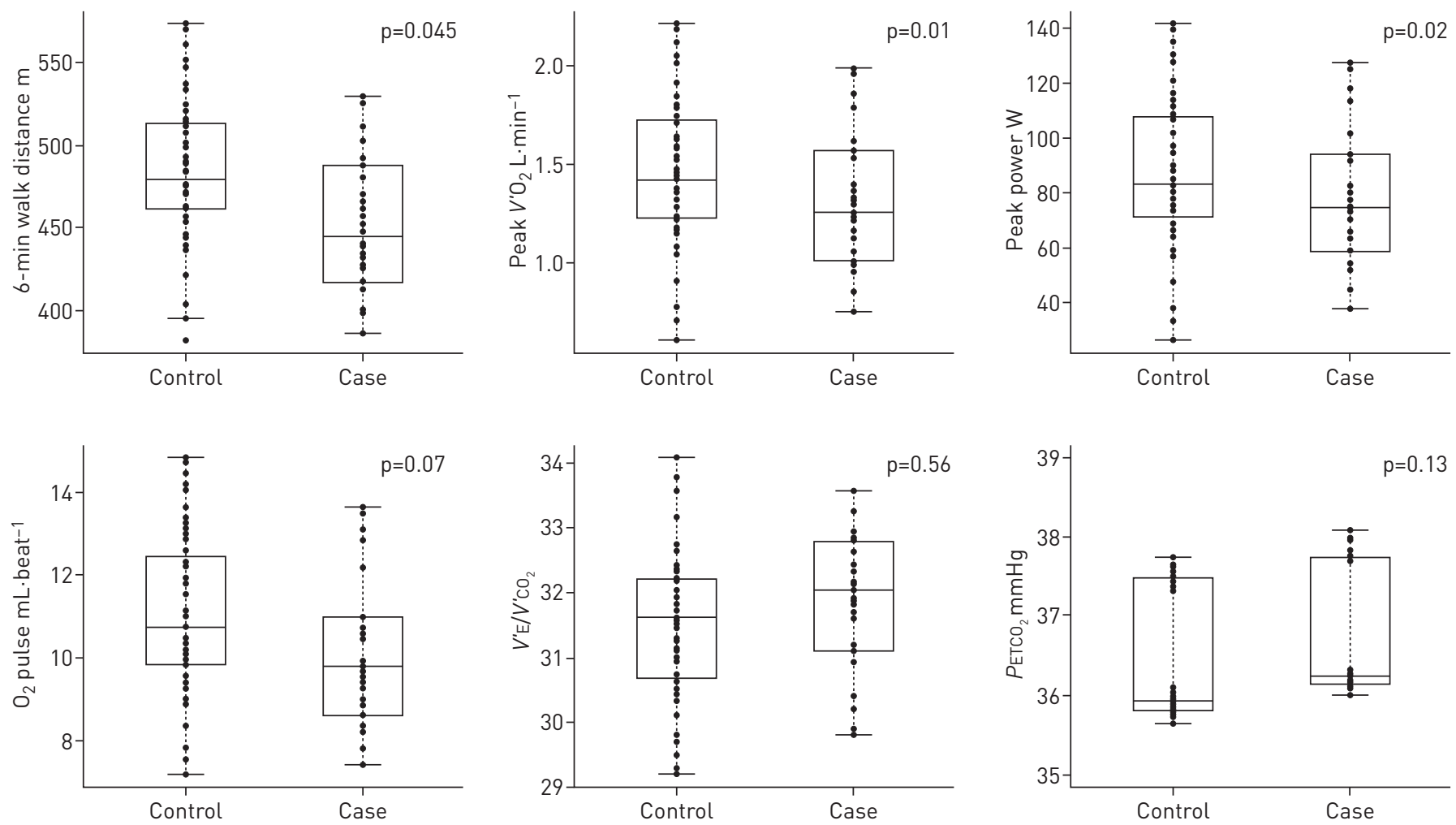

FIGURE 2 Boxplots of exercise capacity at 10.8 years of follow-up in 30 cases with elevated high attenuation area (HAA) (>7.5\%) and 59 controls without elevated HAA on Multi-Ethnic Study of Atherosclerosis (MESA) baseline computed tomography scans. $\mathrm{O}_{2}$ pulse, minute ventilation/CO production $\left(\mathrm{V}^{\prime} \mathrm{E} / \mathrm{V}^{\prime} \mathrm{CO}_{2}\right)$ and end-tidal $\mathrm{CO}_{2}$ tension $\left(\mathrm{PETCO}_{2}\right)$ were measured at peak exercise. Dots represent individual predicted values adjusted for age and sex. Solid horizontal lines represent the median HAA value. Boxes are bound by the upper and lower quartiles. Whiskers extend to 1.5-times the interquartile range. $\mathrm{V}^{\prime} \mathrm{O}_{2}$ : oxygen uptake. 

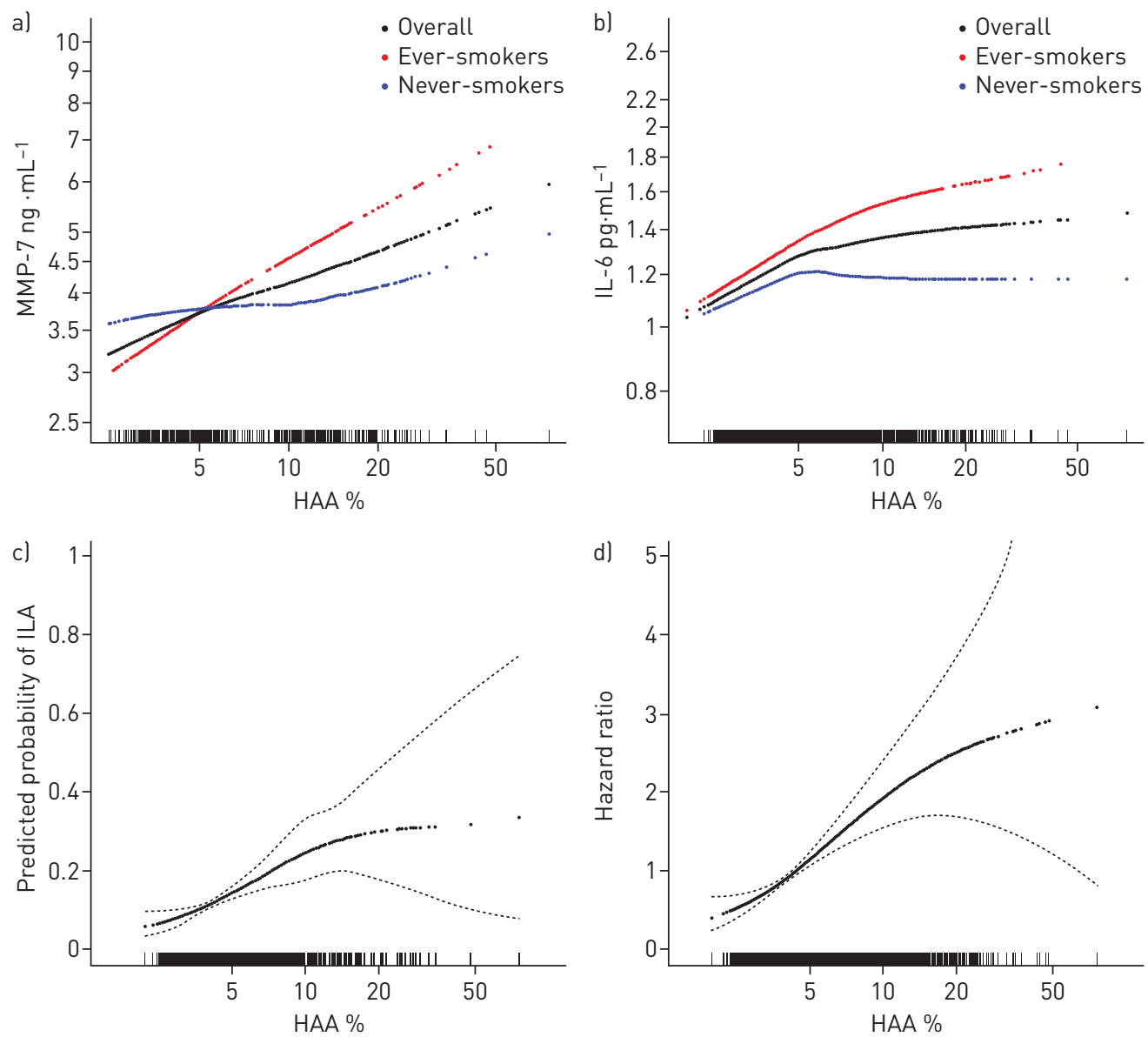

FIGURE 3 Continuous associations between high attenuation area (HAA) and a) matrix metalloproteinase (MMP)-7 (overall and stratified by smoking status) at baseline among 908 MESA participants, b) interleukin (IL)-6 (overall and stratified by smoking status) at baseline among 6621 Multi-Ethnic Study of Atherosclerosis (MESA) participants, c) interstitial lung abnormalities (ILA)) 9.5 years following HAA measurement among 2430 MESA participants and d) mortality over 12.2 years of follow-up among 6808 MESA participants. All models adjusted for age, sex, race/ethnicity, educational attainment, height, body mass index, waist circumference, glomerular filtration rate, study site, $\mathrm{mA}$ dose, total volume imaged lung and percentage emphysema; overall models also adjusted for smoking status and cigarette pack-years. al Overall p-value for association 0.01, $\mathrm{p}$-value for non-linearity 0.37; among ever-smokers, $\mathrm{p}$-value for association <0.001, $\mathrm{p}$-value for non-linearity 0.70 ; among never-smokers, $p$-value for association 0.69 , $p$-value for non-linearity 0.18 ; $p$-value for smoking interaction $<0.001$. b) Overall $p$-value for association $<0.001, p$-value for non-linearity 0.005; among ever-smokers $p$ for association <0.001; $p$-value for non-linearity 0.17 ; among never-smokers $p$-value for association 0.28; $p$-value for non-linearity 0.02 ; $p$-value for smoking interaction 0.11 . c) $p$-value for association <0.001; $p$-value for non-linearity 0.05. d) $p$-value for association $<0.001$. Black points are the overall effect estimate; thin dashed lines are the $95 \%$ confidence bands; red points are the effect estimate among ever-smokers; blue points are the effect estimate among never-smokers. Each point in the graphs and each vertical mark in the rug plot along the $\mathrm{x}$-axis represent one study participant.

capacity, greater odds of ILA at 10-year follow-up and a higher mortality rate. Some of these associations were stronger among ever-smokers compared with never-smokers. These findings support the construct validity of HAA as a novel quantitative phenotype of subclinical lung injury and inflammation that is of biological and physiological importance and that may be a precursor of subclinical ILD.

The associations between HAA and both IL-6 and MMP-7 suggest that HAA identifies areas of interstitial inflammation, extracellular matrix remodelling, and perhaps fibrosis in some cases. IL-6 is a pro-inflammatory cytokine and its serum levels are elevated in acute lung injury [32, 33]. IL-6 has been implicated in chronic obstructive pulmonary disease [34], acute respiratory distress syndrome [35] and certain types of ILD [36]. IL-6 levels have also been linked with reduced FVC percentage in population-based cohorts [37, 38]. IL-6 induces hepatic production of CRP and other downstream inflammatory mediators [12]. While IL-6 levels often correlate with CRP, there are a number of conditions where the levels of the two biomarkers do not track each other [39-41], suggesting that there are additional pathways that regulate these biomarkers, which may explain why we did not see an association 
TABLE 2 Associations between baseline high attenuation areas (HAA) and interstitial lung abnormalities (ILA) at 9.5 years of follow-up in Multi-Ethnic Study of Atherosclerosis (MESA) participants ${ }^{\#}$

\begin{tabular}{|c|c|c|c|c|c|c|c|}
\hline & \multicolumn{4}{|c|}{ HAA } & \multirow{2}{*}{$p$-value for trend } & \multirow[t]{2}{*}{ Overall } & \multirow[t]{2}{*}{ p-value } \\
\hline & Quartile 1 & Quartile 2 & Quartile 3 & Quartile 4 & & & \\
\hline Number with ILA & 61 & 72 & 72 & 101 & & 306 & \\
\hline \multicolumn{6}{|l|}{ OR $(95 \% \mathrm{Cl})$} & \multicolumn{2}{|c|}{$\begin{array}{c}\text { OR }(95 \% \text { CI) per doubling } \\
\text { of HAA }\end{array}$} \\
\hline Adjusted " & Ref & $1.42(0.93-2.17)$ & $1.79(1.11-2.89)$ & 3.07 (1.77-5.33) & $<0.001$ & 1.95 (1.43-2.65) & $<0.001$ \\
\hline \multicolumn{8}{|c|}{$\begin{array}{l}\text { Adjusted, stratified by } \\
\text { smoking status }{ }^{+}\end{array}$} \\
\hline Ever-smokers & Ref & $1.14(0.66-1.99)$ & $1.53(0.84-2.81)$ & $2.31(1.13-4.73)$ & 0.009 & $2.38(1.57-3.60)$ & $<0.001$ \\
\hline Never-smokers & Ref & $2.34(1.18-4.64)$ & $2.55(1.15-5.65)$ & $5.55(2.24-13.77)$ & $<0.001$ & 1.59 (0.99-2.55) & 0.053 \\
\hline
\end{tabular}

TABLE 3 Associations between baseline high attenuation areas (HAA) and mortality over 12.2 years of follow-up in 6808 Multi-Ethnic Study of Atherosclerosis (MESA) participants

\begin{tabular}{|c|c|c|c|c|c|c|c|}
\hline & \multicolumn{4}{|c|}{ HAA } & $\mathrm{p}$-value for trend & Overall & p-value \\
\hline Number at-risk & 1699 & 1699 & 1700 & 1699 & \multicolumn{3}{|c|}{6808} \\
\hline Number of decedents & 252 & 237 & 283 & 321 & \multicolumn{3}{|c|}{1093} \\
\hline $\begin{array}{l}\text { Person-years of } \\
\text { observation }\end{array}$ & 19010 & 18805 & 18844 & 18347 & \multicolumn{3}{|c|}{75006} \\
\hline $\begin{array}{l}\text { Mortality rate per } 1000 \\
\text { person-years }\end{array}$ & 13.3 & 12.6 & 15.0 & 17.5 & \multicolumn{3}{|c|}{14.6} \\
\hline $\operatorname{HR}(95 \% \mathrm{CI})$ & & & & & & \multicolumn{2}{|c|}{$\begin{array}{c}\text { HR }(95 \% \text { CI) per doubling } \\
\text { of HAA }\end{array}$} \\
\hline Model $1^{\#}$ & Ref & $1.15(0.94-1.40)$ & 1.51 (1.23-1.87) & $1.94(1.53-2.46)$ & $<0.001$ & $1.58(1.39-1.79)$ & $<0.001$ \\
\hline Model 2" & Ref & $1.15(0.95-1.41)$ & 1.52 (1.23-1.88) & $1.91(1.51-2.43)$ & $<0.001$ & 1.55 (1.37-1.77) & $<0.001$ \\
\hline \multicolumn{8}{|l|}{$\begin{array}{l}\text { Model 2, stratified by } \\
\text { smoking status }{ }^{+}\end{array}$} \\
\hline \multicolumn{8}{|c|}{$\begin{array}{l}\text { \#: adjusted for age, sex, race/ethnicity, educational attainment, height, body mass index, waist circumference, smoking status, cigarette } \\
\text { pack-years, glomerular filtration rate, study site, mA dose, total volume of imaged lung and percent emphysema. Adjustment for smoking status } \\
\text { and pack-years was excluded from smoking-stratified models. All covariates measured at baseline examination in } 2000-2002 .{ }^{\uparrow}: \text { model } 2 \text { is } \\
\text { additionally adjusted for alcohol use, exercise, coronary artery calcium, diabetes medication use, insulin use, fasting glucose level, hypertension, } \\
\text { antihypertensive medication use, systolic and diastolic blood pressures, cholesterol medication use, total and high-density lipoprotein cholesterol } \\
\text { levels, C-reactive protein level, D-dimer level and history of cancer. }{ }^{+}: \text {- p-value for interaction } 0.56 \text {. }\end{array}$} \\
\hline
\end{tabular}

between HAA and CRP in our study. MMP-7 is thought to play an important role in the normal response to epithelial injury [42]. Epithelial expression of MMP-7 is increased following injury and in the setting of both lung inflammation and fibrosis [18-20,43,44]. We found that the association between HAA and serum MMP-7 (and, to a lesser extent, IL-6) was stronger among ever-smokers, providing support for $\mathrm{HAA}$ as a measure of cigarette smoke-induced alveolar remodelling.

While, in some individuals, HAA might represent hydrostatic pulmonary oedema, pulmonary infection, malignant or pre-malignant lesions, or basilar atelectasis due to obesity or incomplete inspiration, the 
totality of our findings suggests that HAA identifies abnormalities in lung structure with biological and physiological characteristics of subclinical ILD. We found no evidence of strong confounding by cardiac function, BMI, waist circumference or total volume of imaged lung. MESA participants were generally well at the time of enrolment and were rescheduled or excluded if they had an acute infectious process or clinically diagnosed heart failure, respectively. The overall good health of MESA participants decreases the likelihood that HAA reflects the participants' inability to perform a full inspiratory manoeuvre. A particular strength of our study lies in the consistency of expected changes across a number of radiological, biological and physiological measurements as well as evidence of effect modification by smoking, which would not be expected if HAA represented left ventricular filling pressures or obesity.

Others have used alternative definitions of HAA ( -700 to $0 \mathrm{HU},-700$ to $-500 \mathrm{HU}$ ) [45-47], histogram characteristics, and other automated methods [48-51], including texture-based methods [52], to identify and characterise clinically evident ILD, but not subclinical changes. Recent studies have examined the associations between visually identified ILA and mortality [9], but ours is the first study of HAA as a quantitative measure of lung injury and inflammation that is associated with a higher rate of death in community dwelling adults sampled without regard to lung disease, symptoms or smoking status. Our results were consistent across the entire spectrum of HAA, and were particularly strong even after exclusion of individuals above the 90th percentile of HAA, a group who might have clinically identifiable ILD. The majority of participants had HAA involving less than $10 \%$ of imaged lung, suggesting that even small amounts of increased lung attenuation, which might be difficult to detect with the human eye, may identify individuals at risk for ILD. If validated, investigators may be able to use HAA to study causes of early injury, inflammation, and extracellular matrix remodelling of the lung, even before a radiologist identifies the presence of interstitial lung abnormalities. Such studies might allow the identification of novel risk factors and biological pathways for ILD in humans, setting the stage for future clinical trials aimed at primary and secondary prevention of ILD [53].

There are several limitations to our study. First, we lack pathological correlates of disease against which HAA can be validated. This was a study of subclinical disease in community-dwelling adults unselected for any respiratory symptoms and, therefore, with no available pathological samples. However, we also measured HAA in a second cohort of adults with clinically diagnosed ILD, most of whom had IPF diagnosed by ATS criteria, and we report HAA values that are significantly higher than those of MESA participants. Second, HAA was assessed on cardiac CTs, which do not image the entire lung, but which have been shown to be useful in examining emphysema $[31,54,55]$. We have previously shown that HAA on cardiac CT agrees strongly with HAA on full-lung CT scans [10]. Third, we report cross-sectional analyses between HAA and biomarkers, which limits inferences about any causal relationships. However, prior studies have firmly established MMP-7 as a biomarker of lung injury and ILD, IL-6 has been well described in the literature as a marker of inflammation, and our ILA and mortality analyses take place over a median follow-up time of 10-12 years. Fourth, we lacked baseline assessment of ILA and therefore can only report the period cross-sectional associations between HAA and ILA. Given the long follow-up period in our study and the 10-year difference between assessment of HAA and ILA, we believe that at least in some cases, HAA precedes the development of ILA. However, the odds ratios we report should not be interpreted as incidence rate ratios.

In summary, greater volumes of increased lung attenuation measured using quantitative CT densitometry are associated with higher serum IL-6 and MMP-7 levels, reductions in FVC, an increased odds of ILA and an increased rate of all-cause mortality independent of potential confounding factors among communitydwelling adults. Our data suggest that HAA is a novel quantitative measure of subclinical lung injury, inflammation and possibly early extracellular matrix remodelling that may be a CT-based risk factor for the development of interstitial lung abnormalities and disease. Future studies should focus on factors that influence HAA in at-risk groups, such as smokers or older adults and should strive to include HAA in clinical prediction models for incident ILD. Such studies will lead to clinical trials of interventions to prevent ILD.

\section{Acknowledgements}

The authors thank the other investigators, the staff and the participants of the MESA study for their valuable contributions. A full list of participating MESA investigators and institutions can be found at http://www.mesa-nhlbi.org.

Anna J. Podolanczuk, Elizabeth C. Oelsner, David J. Lederer, R. Graham Barr, and Steven M. Kawut conceived and designed the study. Anna J. Podolanczuk, Elizabeth C. Oelsner, R. Graham Barr, Hilary F. Armstrong, Robert C. Basner, Matthew N. Bartels, Jason D. Christie, Karen Hinckley Stukovsky, Joel D. Kaufman, Scott M. Palmer, Russell P. Tracy, Kayleen Williams, and David J. Lederer acquired the data. Anna J. Podolanczuk, Elizabeth C. Oelsner, R. Graham Barr, Eric A. Hoffman, John H.M. Austin, Robert C. Basner, Matthew N. Bartels, Karen Hinckley Stukovsky, P. Hrudaya Nath, John D. Newell, Dan Rabinowitz, Jessica L. Sell, Jered Sieren, Sushil K. Sonavane, Russell P. Tracy, Jubal R. Watts, Kayleen Williams, and David J. Lederer analysed the data. All authors contributed to interpretation of data. Anna J. Podolanczuk drafted the initial manuscript. All authors revised the manuscript for important intellectual content and approved the final version of the manuscript. All authors agree to be accountable for all aspects of the work 


\section{References}

1 Selman M, Pardo A. Alveolar epithelial cell disintegrity and subsequent activation: a key process in pulmonary fibrosis. Am J Respir Crit Care Med 2012; 186: 119-121.

2 Zoz DF, Lawson WE, Blackwell TS. Idiopathic pulmonary fibrosis: a disorder of epithelial cell dysfunction. Am J Med Sci 2011; 341: 435-438.

3 Rosas IO, Ren P, Avila NA, et al. Early interstitial lung disease in familial pulmonary fibrosis. Am J Respir Crit Care Med 2007; 176: 698-705.

4 Doyle TJ, Hunninghake GM, Rosas IO. Subclinical interstitial lung disease: why you should care. Am J Respir Crit Care Med 2012; 185: 1147-1153.

5 Kropski JA, Pritchett JM, Zoz DF, et al. Extensive phenotyping of individuals at-risk for familial interstitial pneumonia reveals clues to the pathogenesis of interstitial lung disease. Am J Respir Crit Care Med 2014; 191: 417-426.

6 Washko GR, Hunninghake GM, Fernandez IE, et al. Lung volumes and emphysema in smokers with interstitial lung abnormalities. N Engl J Med 2011; 364: 897-906.

7 Doyle TJ, Washko GR, Fernandez IE, et al. Interstitial lung abnormalities and reduced exercise capacity. Am J Respir Crit Care Med 2012; 185: 756-762.

8 Hunninghake GM, Hatabu H, Okajima $\mathrm{Y}$, et al. MUC5B promoter polymorphism and interstitial lung abnormalities. N Engl J Med 2013; 368: 2192-2200.

9 Putman RK, Hatabu H, Araki T, et al. Association between interstitial lung abnormalities and all-cause mortality. JAMA 2016; 315: 672-681.

10 Lederer DJ, Enright PL, Kawut SM, et al. Cigarette smoking is associated with subclinical parenchymal lung disease: the Multi-Ethnic Study of Atherosclerosis (MESA)-lung study. Am J Respir Crit Care Med 2009; 180: 407-414.

11 Kliment CR, Araki T, Doyle TJ, et al. A comparison of visual and quantitative methods to identify interstitial lung abnormalities. BMC Pulm Med 2015; 15: 134.

12 Gauldie J, Richards C, Harnish D, et al. Interferon beta 2/B-cell stimulatory factor type 2 shares identity with monocyte-derived hepatocyte-stimulating factor and regulates the major acute phase protein response in liver cells. Proc Natl Acad Sci USA 1987; 84: 7251-7255.

13 Kushner I, Feldmann G. Control of the acute phase response. Demonstration of C-reactive protein synthesis and secretion by hepatocytes during acute inflammation in the rabbit. J Exp Med 1978; 148: 466-477.

14 Greene KE, Wright JR, Steinberg KP, et al. Serial changes in surfactant-associated proteins in lung and serum before and after onset of ARDS. Am J Respir Crit Care Med 1999; 160: 1843-1850.

15 Ohnishi H, Yokoyama A, Kondo K, et al. Comparative study of KL-6, surfactant protein-A, surfactant protein-D, and monocyte chemoattractant protein-1 as serum markers for interstitial lung diseases. Am J Respir Crit Care Med 2002; 165: 378-381.

16 Greene KE, King TE Jr, Kuroki Y, et al. Serum surfactant proteins-A and -D as biomarkers in idiopathic pulmonary fibrosis. Eur Respir J 2002; 19: 439-446.

17 Kinder BW, Brown KK, McCormack FX Jr, et al. Serum surfactant protein-A is a strong predictor of early mortality in idiopathic pulmonary fibrosis. Chest 2009; 135: 1557-1563.

18 Richards TJ, Kaminski N, Baribaud F, et al. Peripheral blood proteins predict mortality in idiopathic pulmonary fibrosis. Am J Respir Crit Care Med 2012; 185: 67-76.

19 Rosas IO, Richards TJ, Konishi K, et al. MMP1 and MMP7 as potential peripheral blood biomarkers in idiopathic pulmonary fibrosis. PLoS Med 2008; 5: e93.

20 Zuo F, Kaminski N, Eugui E, et al. Gene expression analysis reveals matrilysin as a key regulator of pulmonary fibrosis in mice and humans. Proc Natl Acad Sci USA 2002; 99: 6292-6297.

21 Bild DE, Bluemke DA, Burke GL, et al. Multi-ethnic study of atherosclerosis: objectives and design. Am Epidemiol 2002; 156: 871-881.

22 Carr JJ, Nelson JC, Wong ND, et al. Calcified coronary artery plaque measurement with cardiac CT in population-based studies: standardized protocol of Multi-Ethnic Study of Atherosclerosis (MESA) and Coronary Artery Risk Development in Young Adults (CARDIA) study. Radiology 2005; 234: 35-43.

23 Hoffman EA, Jiang R, Baumhauer $\mathrm{H}$, et al. Reproducibility and validity of lung density measures from cardiac CT Scans-The Multi-Ethnic Study of Atherosclerosis (MESA) Lung Study. Acad Radiol 2009; 16: 689-699.

24 Gevenois PA, de Maertelaer V, De Vuyst P, et al. Comparison of computed density and macroscopic morphometry in pulmonary emphysema. Am J Respir Crit Care Med 1995; 152: 653-657.

25 Miller MR, Hankinson J, Brusasco V, et al. Standardisation of spirometry. Eur Respir J 2005; 26: 319-338

26 Hankinson JL, Kawut SM, Shahar E, et al. Performance of American Thoracic Society-recommended spirometry reference values in a multiethnic sample of adults: the multi-ethnic study of atherosclerosis (MESA) lung study. Chest 2010; 137: 138-145.

27 ATS Committee on Proficiency Standards for Clinical Pulmonary Function Laboratories. ATS statement: guidelines for the six-minute walk test. Am J Respir Crit Care Med 2002; 166: 111-117.

28 Couper D, LaVange LM, Han M, et al. Design of the Subpopulations and Intermediate Outcomes in COPD Study (SPIROMICS). Thorax 2014; 69: 491-494.

29 Hoffman EA, Ahmed FS, Baumhauer H, et al. Variation in the percent of emphysema-like lung in a healthy, nonsmoking multiethnic sample. The MESA lung study. Ann Am Thorac Soc 2014; 11: 898-907.

30 Washko GR, Lynch DA, Matsuoka S, et al. Identification of early interstitial lung disease in smokers from the COPDGene Study. Acad Radiol 2010; 17: 48-53.

31 Oelsner EC, Hoffman EA, Folsom AR, et al. Association between emphysema-like lung on cardiac computed tomography and mortality in persons without airflow obstruction: a cohort study. Ann Intern Med 2014; 161: 863-873.

32 Kolb M, Margetts PJ, Anthony DC, et al. Transient expression of IL-1beta induces acute lung injury and chronic repair leading to pulmonary fibrosis. J Clin Invest 2001; 107: 1529-1536.

33 Zhang H, Neuhöfer P, Song L, et al. IL-6 trans-signaling promotes pancreatitis-associated lung injury and lethality. J Clin Invest 2013; 123: 1019-1031.

34 Garcia-Rio F, Miravitlles M, Soriano JB, et al. Systemic inflammation in chronic obstructive pulmonary disease: a population-based study. Respir Res 2010; 11: 63 
Hoeboer SH, Groeneveld AB, van der Heijden M, et al. Serial inflammatory biomarkers of the severity, course and outcome of late onset acute respiratory distress syndrome in critically ill patients with or at risk for the syndrome after new-onset fever. Biomark Med 2015; 9: 605-616.

36 Shahar I, Fireman E, Topilsky M, et al. Effect of IL-6 on alveolar fibroblast proliferation in interstitial lung diseases. Clin Immunol Immunopathol 1996; 79: 244-251.

37 Chang SS, Vaz Fragoso CA, Van Ness PH, et al. Association between combined interleukin-6 and C-reactive protein levels and pulmonary function in older women: results from the Women's Health and Aging Studies I and II. J Am Geriatr Soc 2011; 59: 113-119.

38 Thorleifsson SJ, Margretardottir OB, Gudmundsson G, et al. Chronic airflow obstruction and markers of systemic inflammation: results from the BOLD study in Iceland. Respir Med 2009; 103: 1548-1553.

39 Lee BT, Ahmed FA, Hamm LL, et al. Association of C-reactive protein, tumor necrosis factor-alpha, and interleukin-6 with chronic kidney disease. BMC Nephrol 2015; $16: 77$.

40 Czarkowska-Paczek B, Bartlomiejczyk I, Gabrys T, et al. Lack of relationship between interleukin-6 and CRP levels in healthy male athletes. Immunol Lett 2005; 99: 136-140.

41 Bermudez EA, Rifai N, Buring J, et al. Interrelationships among circulating interleukin-6, C-reactive protein, and traditional cardiovascular risk factors in women. Arterioscler Thromb Vasc Biol 2002; 22: 1668-1673.

42 Gharib SA, Altemeier WA, Van Winkle LS, et al. Matrix metalloproteinase-7 coordinates airway epithelial injury response and differentiation of ciliated cells. Am J Respir Cell Mol Biol 2013; 48: 390-396.

43 Villar J, Cabrera-Benítez NE, Ramos-Nuez A, et al. Early activation of pro-fibrotic WNT5A in sepsis-induced acute lung injury. Crit Care 2014; 18: 568.

44 Dunsmore SE, Saarialho-Kere UK, Roby JD, et al. Matrilysin expression and function in airway epithelium. J Clin Invest 1998; 102: 1321-1331.

45 Shin KE, Chung MJ, Jung MP, et al. Quantitative computed tomographic indexes in diffuse interstitial lung disease: correlation with physiologic tests and computed tomography visual scores. J Comput Assist Tomogr 2011; 35: $266-271$.

46 Matsuoka S, Yamashiro T, Matsushita S, et al. Quantitative CT evaluation in patients with combined pulmonary fibrosis and emphysema: correlation with pulmonary function. Acad Radiol 2015; 22: 626-631.

47 Matsuoka S, Yamashiro T, Matsushita S, et al. Morphological disease progression of combined pulmonary fibrosis and emphysema: comparison with emphysema alone and pulmonary fibrosis alone. J Comput Assist Tomogr 2015; 39: $153-159$.

48 Maldonado F, Moua T, Rajagopalan S, et al. Automated quantification of radiological patterns predicts survival in idiopathic pulmonary fibrosis. Eur Respir J 2014; 43: 204-212.

49 Noor NM, Than JC, Rijal OM, et al. Automatic lung segmentation using control feedback system: morphology and texture paradigm. J Med Syst 2015; 39: 22.

50 Moon JW, Bae JP, Lee HY, et al. Perfusion- and pattern-based quantitative CT indexes using contrast-enhanced dual-energy computed tomography in diffuse interstitial lung disease: relationships with physiologic impairment and prediction of prognosis. Eur Radiol 2016; 26: 1368-1377.

51 Depeursinge A, Chin AS, Leung AN, et al. Automated classification of usual interstitial pneumonia using regional volumetric texture analysis in high-resolution computed tomography. Invest Radiol 2015; 50: 261-267.

$52 \mathrm{Xu}$ Y, van Beek EJ, Hwanjo Y, et al. Computer-aided classification of interstitial lung diseases via MDCT: 3D adaptive multiple feature method (3D AMFM). Acad Radiol 2006; 13: 969-978.

53 Lederer DJ. Secondary prevention of idiopathic pulmonary fibrosis: catching the horse still in the barn. Am J Respir Crit Care Med 2012; 185: 697-699.

54 Barr RG, Bluemke DA, Ahmed FS, et al. Percent emphysema, airflow obstruction, and impaired left ventricular filling. N Engl J Med 2010; 362: 217-227.

55 Chapman KR, Burdon JG, Piitulainen E, et al. Intravenous augmentation treatment and lung density in severe alpha1 antitrypsin deficiency (RAPID): a randomised, double-blind, placebo-controlled trial. Lancet 2015; 386: 360-368. 